\title{
Fuzzy Multi-Agent Based Voltage and Reactive Power Control
}

\author{
Bessie Monchusi ${ }^{1}$, Adedayo Yusuff ${ }^{1}$, Josiah Munda ${ }^{1}$, Adisa Jimoh ${ }^{1}$ \\ ${ }^{1}$ Department of Electrical Engineering \\ Tshwane University of Technology \\ Private Bag X680, 0001 Pretoria (South Africa) \\ Phone/Fax number: +0027 12382 4824, e-mail: MonchusiBB@tut.ca.za, YusuffAA@ tut.ca.za, MundaJL@tut.ca.za, \\ JimohAA@tut.ca.za
}

\begin{abstract}
Variations in load and generation profiles cause overvoltages leading to equipment insulation failure and undervoltages, which impact voltage stability margin. Voltage and reactive power control aims to keep the voltage profiles within desired limits and reduce power losses. In this paper multi-agent system based on Java technology and adaptive fuzzy logics algorithms are implemented to control voltage and reactive power. Agents communicate with each other using Foundation for Intelligent Physical Agents (FIPA) to be able to achieve their objectives. A fuzzy agent controls the voltage at the busbars. Multi-agent systems make it possible to do three hierarchal voltage control in distribution systems.
\end{abstract}

\section{Key words}

Fuzzy Agent, Java Agent Development (JADE), MultiAgent Systems, Voltage Control.

\section{Introduction}

"We cannot solve problems by using the same kind of thinking we used when we created them." (Albert Einstein). This was supported by the illustration for a need of different voltage control methods after major power system blackouts throughout the world in 2003. Furthermore, the distribution network is becoming populated with DGs and thus changing the character from uni-directional to bi-directional flow. The voltage and reactive power equipment are operated with the assumption that the voltage decreases along the feeder. Distributed generators alter the feeder voltage profiles and this will affect the way voltage is controlled in distribution systems [1].This brings a need for new control methods.

In conventional distribution systems, voltage and reactive power control is normally done by on-load tap changers (OLTC) and switched shunt capacitors. OLTC keep the substation secondary bus voltage constant by adjusting the tap position. The switched shunt capacitor is used to compensate the reactive power demand and thereby decrease the voltage drop. The problem with the conventional methods is that power losses may not be minimized. DGs are not involved in voltage control although they are faster in operation than the OLTC and mechanically switched shunt capacitors. If DGs are involved in the voltage control, the voltage control will be similar to the transmission's one where it is deployed in three hierarchical levels [2].

SVCs which use local control method have been used for voltage control previously. The disadvantage of this method for voltage control is that the control performance of SVC degrades after great changes in power network configuration.

Traditional voltage control methods like decreasing of OLTC set point voltage, altering the capacitor control or limiting the DG size according to the worst case scenario are all local control methods which will not be capable of fast voltage regulation in a decentralized generation scenario. The effect of the DGs to increase voltage at their terminals can be harnessed to contribute to voltage control.

In using the DG in coordinated voltage control, three level hierarchical level of control can be used in distribution systems. Primary control will be performed by DGs, secondary control by locally operated OLTCs and switched capacitors and the tertiary control by remotely adjusting DG, OLTC and substation capacitors. To ensure that fast reactive power reserves near the bus where voltage collapse occurs because of a large disturbance, the multi-agent technology can be applied to Secondary Voltage Control (SVC) of the power system. The primary voltage controllers and the SVCs will be represented as a set of executive agents and co-ordination 
agent working either independently or co-ordinated to control the system wide voltage level.

The objective of the paper is to minimize voltage deviations from the given reference values in buses. This is achieved by designing a fast and coordinated voltage and reactive power control system with three level hierarchical structure which is decentralized and applies the fuzzy agent for controlling the voltage and reactive power.

The proposed fuzzy algorithm is used to find the setting of tap positions of tap changing transformers to optimize the voltage profile.

The article is structured as follows: Section 2 provides an overview of the multi-agent system; in Section 3 Fuzzy Agent is discussed; Section 4 discusses the voltage control algorithm. Section 5 discusses simulations and results. The paper is concluded in Section 6.

\section{Multi-Agent System (MAS)}

The main element of an MAS system is the agent. The agent is either a physical entity which can be a microsource or a controllable load, or a virtual entity, which is a piece of software that coordinates the agents. An agent is capable of changing its environment. They communicate with each other and this is part of their capability of acting in the environment [3].

An agent has certain behaviour and tends to certify certain objectives using its resources. The following agents are used according to their objectives. Agent Management System (AMS), Busbar Agents (BUS), Fuzzy Agent and Execution Agent (EA).

Foundation for Intelligent Physical Agents (FIPA) is used as a communication language between agents. A web service written in Microsoft.Net framework with $\mathrm{C}++$ and running on Microsoft Internal Information services (ITS). It is used to interface MAS and simulation software Java Agent Development (JADE) is used as a platform for developing agents and implementing communication in the system [4].

The multi-agent system architecture and the division of responsibilities between the individual agents, has been built on the work in [5]. An MAS in a hierarchical way shown in Figure 1 is designed with a Busbar Agent located at each bus. A BUS agent is responsible for measuring the voltage at each busbar. The control agent reads the voltage measurements from the BUS agent. It further calculates the voltage deviation and determines if it is within the required limitations. If there is no violation of the limits, no action is taken. In the event of any violation, the CA determines which action needs to be taken to bring the voltage within the required value. The instructions are sent to the EA which ensures that the voltage controlling equipment executes the given instructions.

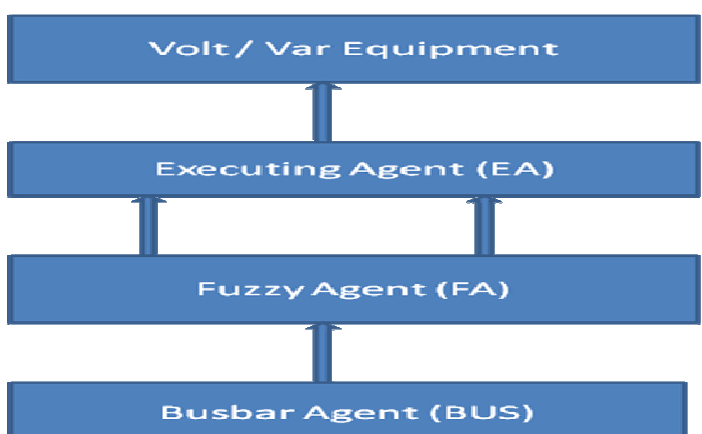

Fig. 1. The layered structure of the multi-agent system and the control with the data flow between them.

\section{Fuzzy Logics}

The learning algorithms used are developed through a fuzzy agent which is based on the fuzzy logics controller concept. The objective function and constraints are formulated in terms of fuzzy sets. The objective is to keep the voltage profile between 0.95 and 1.05 p.u. If the voltage is outside these limits the fuzzy agent issues instructions for the voltage control equipment to rectify the voltage. In cases where the voltage profile is normal, the MAS optimizes the voltage deviation from the selected reference value.

Fuzzy logics make it possible to develop an adaptive and intelligent control system [7] for controlling and monitoring voltage on the secondary side of the transformer. This control is done centrally by the fuzzy agent instead of having a fuzzy logic controller at each busbar. The Mamdani type inference system with the centroid method for deffuzzification is applied. The fuzzy agent operation is shown below using the Matlab fuzzy logic in Figures 2-5. The fuzziness of the controller is characterized by voltage deviation magnitude and voltage deviation. Voltage deviation is calculated as the difference between the reference voltage and the busbar voltage. The voltage deviation magnitude membership is triangular with values under 0.05 assigned to low and values above 0.04 to high as shown in Figure 2. The magnitude of the voltage deviation must be kept at 0.05 or less.
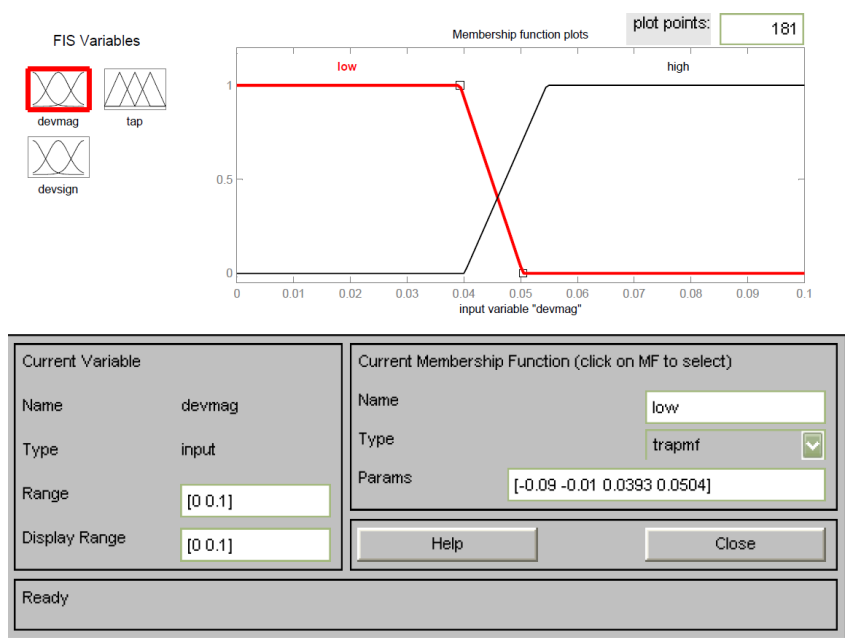

Fig. 2. Membership function for voltage deviation magnitude 
The voltage deviation sign is negative if the reference voltage is lower than the busbar voltage and positive if the condition is the opposite. Figure 3 shows the membership of the voltage deviation sign. The values below zero are assigned to the negative membership, between -0.02 and 0.02 , neutral and positive membership to numbers above zero.

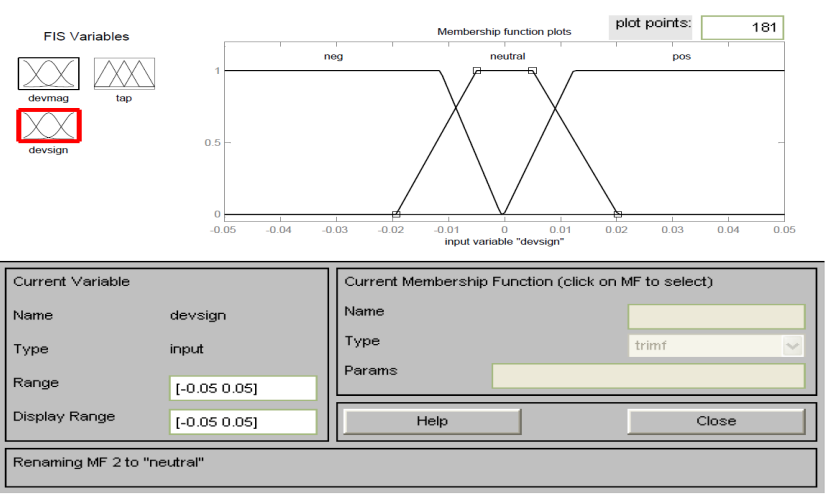

Fig. 3. Membership function for voltage deviation sign

In Figure 4 membership functions are allocated for the direction in which the OLTC must tap to adjust the voltage. The tap changer must step up for values from 0.02 and below and step down from 0.02 and above. For any value between -0.02 and 0.02 there is no tapping allowed, the tap changer must stay in the position where it is.

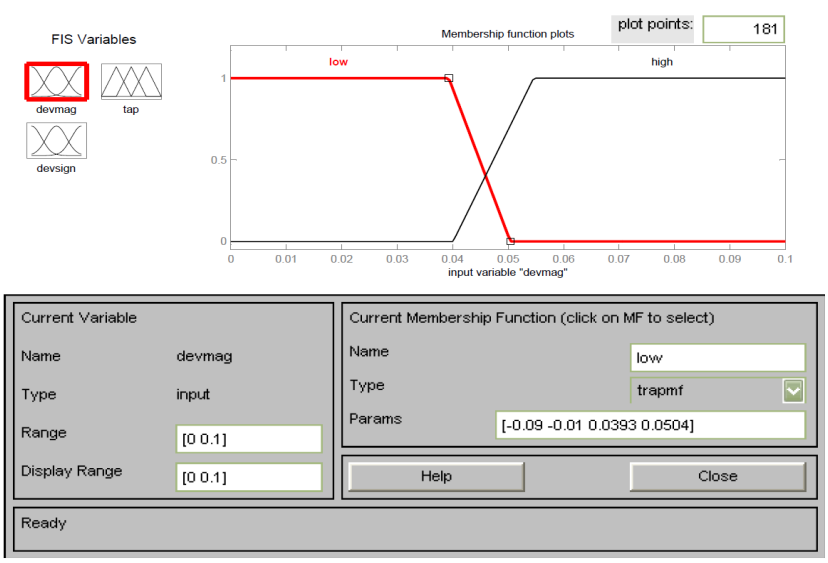

Fig. 4. Membership function for OLTC tapping

The rules of the fuzzy logic controller are shown in Figure 5 :

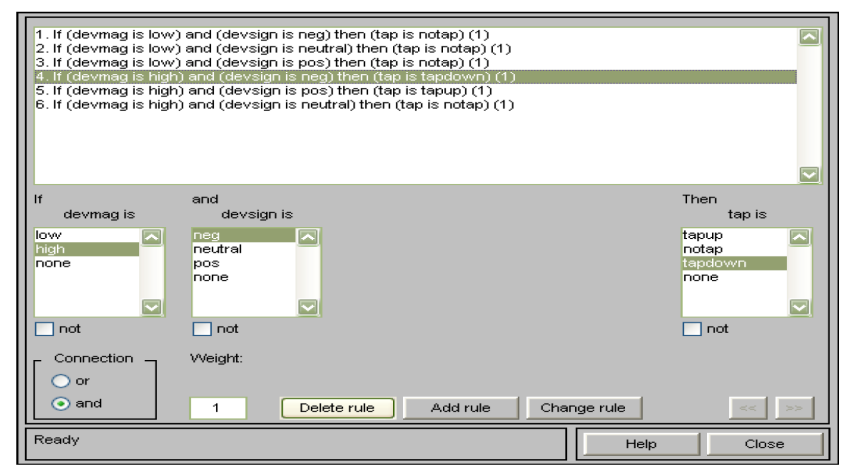

Fig. 5. Fuzzy controller rules
An example of rule number four is shown in figure 6. In this case, the magnitude of the voltage deviation is high; meaning that it is higher than the desired 0.05. The voltage at the busbar is higher than the reference voltage thereby resulting in the deviation sign being negative. The OLTC therefore has to tap down to bring the voltage deviation within the required value.
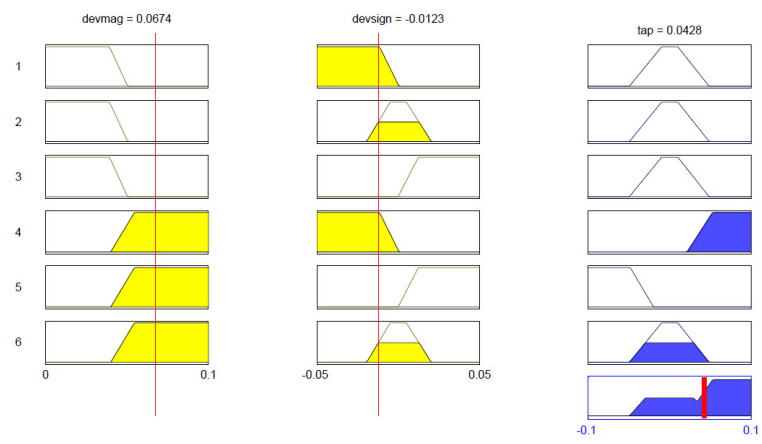

\begin{tabular}{|c|c|c|c|c|c|c|c|}
\hline Input: [0.06743-0.01227] & ||Plot points: & 101 & Move: & left & right & down & up \\
\hline Ready & & & & Help & & Close & \\
\hline
\end{tabular}

Fig. 6. Rule 4 illustration

\section{Voltage Control}

In the voltage control method, the DGs perform the primary voltage control by keeping its terminal voltage equal to the DG reference voltage. The secondary voltage is performed by the capacitor and the OLTC. Tertiary control is performed by developing a short term operational planning, in order to remotely adjust the DG, OLTC and substation capacitors, based on a specification.

The secondary voltage control is the most import in hierarchical voltage control system, for dynamic performance improvement of the power system. Its aim is to realize reactive power production-consumptionbalance. Fast and effective reactive power support is needed for a faulted bus from reactive power reserves. The SVC and the CSVC do not obtain satisfactory results in this regard. To overcome the shortcomings of the conventional SVC system, the multi-agent technology can be applied to the SVC.

Voltage and reactive power equipment in distribution systems are mostly operated based on an assumption that the voltage decreases along the feeder.

The voltage drop along the feeder with a DG generating active and reactive power can be approximated as [1]:

$$
v_{1}^{-} v_{2}=\frac{R\left(P_{L}-P_{G}\right)+X\left(Q_{L}-Q_{G}\right)}{V_{2}}
$$

The objective of the voltage and reactive power control is to minimize deviations of voltages from given reference values in some buses. The presence of DGs controlling the voltage, the number of OLTC operations and voltage fluctuations decreases resulting in the reduction of 
voltage fluctuations. These objectives can be formulated as follows [4]:

$$
\sum\left(w_{p i}\left\|v_{i}-v_{r e f}\right\|^{2}+w_{p i}\left\|\frac{Q_{i}}{Q_{r e f}}\right\|^{2}\right)
$$

$$
\Delta V_{\min } \leq \Delta V_{k, i} \leq \Delta V_{\max }
$$

where Wpi is the weighting coefficient of bus $\mathrm{i}, \mathrm{Vi}$ and $\mathrm{Vi}$, ref are the voltage and reference voltage at bus $i$, Wqi is the reactive power weighting coefficient and (Qi/Qi, ref) indicates the percentage of used reactive resource in bus $i$. The weightings in this objective function can be adjusted based on the importance of each term in the control policy. The MAS will focus on keeping a generator's voltages at reference values or keeping reactive power reserves available. $\Delta V \min =$ minimum deviation between two buses. and $\Delta \mathrm{Vmax}=$ maximum deviation between two buses.

In this study, the CA will be DGs and they will be used to control the voltage magnitude and reactive power reserves. As mentioned before, these intelligent servicing agents can learn and be controlled to provide better and faster performance during power system disturbances.

\section{Simulations}

The power system is simulated in MATLAB. For linking MATLAB to JADE, a web service is written in Microsoft.Net framework with the $\mathrm{C}++$ programming language and is run on Microsoft Internet Information Services (IIS). Two main methods ('Get Status' and 'Set Status') are implemented for reading status from the power system and setting control actions to it by using the Contract Net Protocol (CNP) and the Web Services Description Language (WSDL). Macsim can provide applications running on disparate operating systems and development platforms to communicate with each other. MATLAB also can act as a Macsim client by sending requests to a server and handling the responses.

To illustrate the performance and support the feasibility of the proposed control scheme in practical power systems, a case study based on a $25 \mathrm{kV}$ distribution power system with a 9-MW wind farm consisting of six 1.5 MW wind turbines shown in Figure 7 is used. The wind farm supplies power to a $60 \mathrm{~Hz} 120-\mathrm{kV}$ grid through a $30-\mathrm{km}, 25-\mathrm{kV}$ feeder. A 2300V, 2-MVA plant consisting of a motor load (1.68 MW induction motor at $0.93 \mathrm{PF}$ ) and of a $200-\mathrm{kW}$ resistive load is connected on the same feeder at bus B25. The wind turbine and the motor load have a protection system monitoring voltage, current and machine speed. The DC link voltage of the DFIG is also monitored. A single phase-to-ground fault is applied on the $25-\mathrm{kV}$ line at B25 bus. Figure 8 shows the results of the operation of the power system for a period of 50s without any control applied to it.

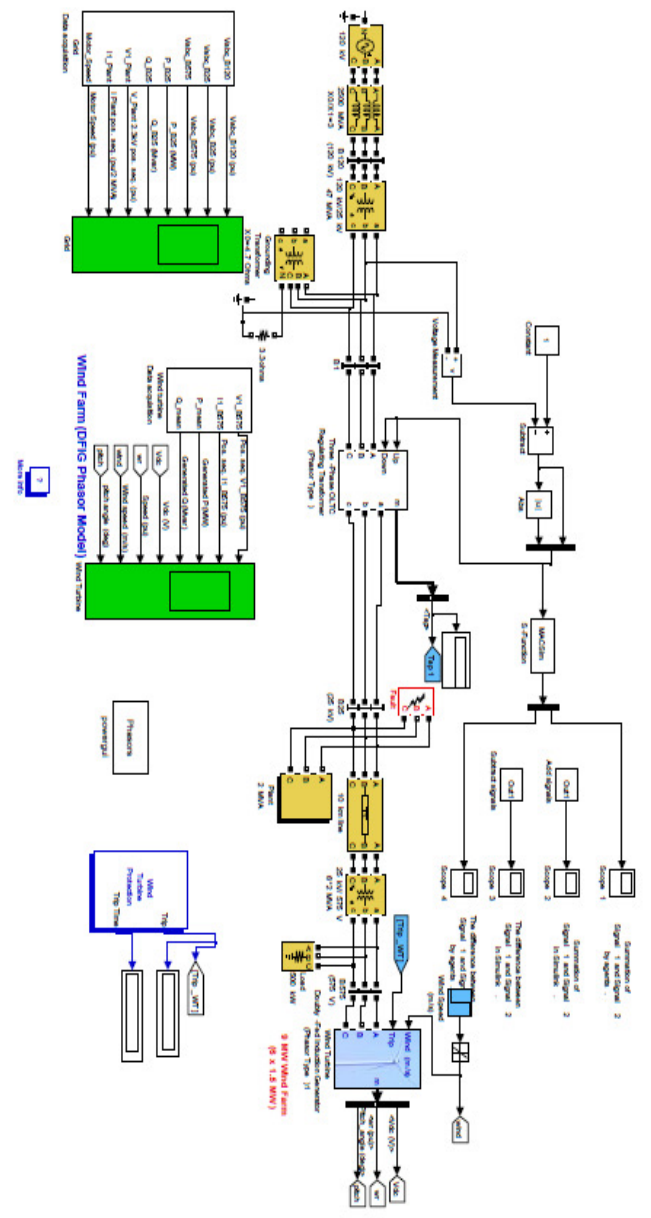

Fig. 7. Single line diagram of the power system

The impact of a voltage sag resulting from a remote fault on the $120-\mathrm{kV}$ system at $\mathrm{t}=5 \mathrm{~s}$ is shown in Figure 8. A voltage drop lasting $0.5 \mathrm{~s}$ occurs at $\mathrm{t}=5 \mathrm{~s}$.

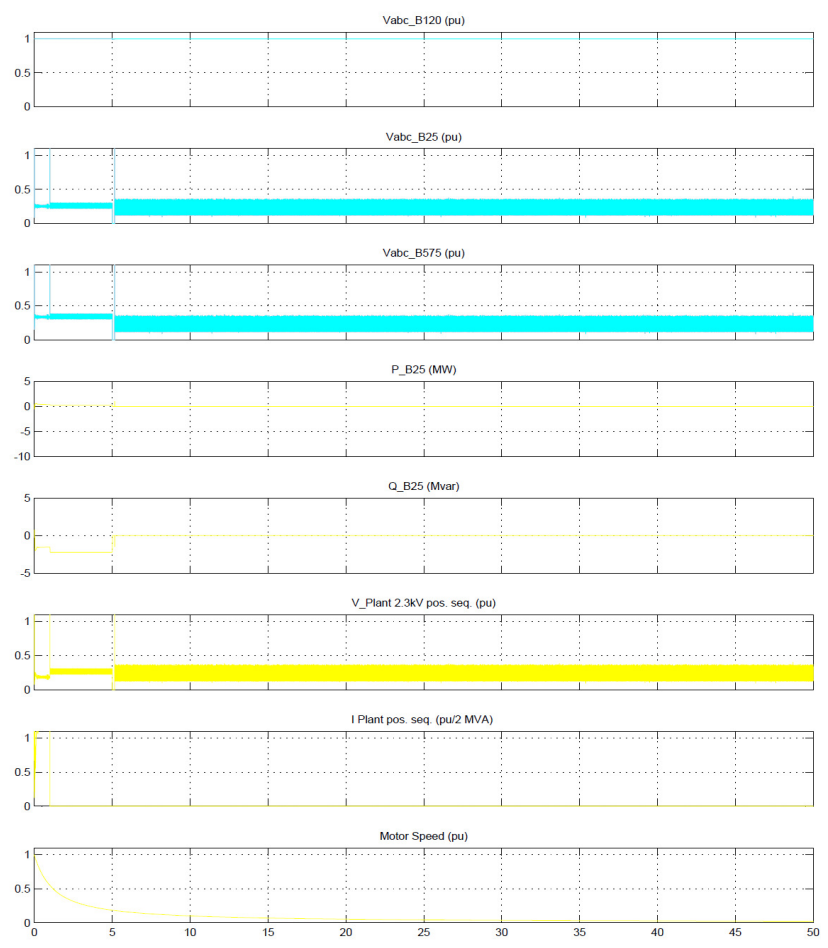

Fig. 8. Results of the operation of the power system for $50 \mathrm{~s}$ with controls. 
On B120 bus the MAS system keeps the plant voltage above the 0.9 p.u. protection threshold during the voltage sag.

\section{Conclusion}

Voltage and reactive power control algorithm is developed using Multi-Agent System and Fuzzy logics. The power system is simulated in MATLAB. Java Agent Development (JADE) is used as a platform for developing agents and implementing communication in the system. For linking MATLAB to JADE, a web service is written in Microsoft.Net framework with the $\mathrm{C}++$ programming language and is run on Microsoft Internet Information Services (IIS). Foundation for Intelligent Physical Agents (FIPA) is used as a communication language between agents. Simulations are done in Matlab on the $25 \mathrm{kV}$ distribution power system with a 9-MW wind farm consisting of six 1.5 MW wind turbines supplying power to a $60 \mathrm{~Hz} 120-\mathrm{kV}$ grid through a $30-\mathrm{km}, 25-\mathrm{kV}$ feeder.

\section{References}

[1] B. Awad, J. Wu, N. Jenkins "Agent based control of Virtual Power Plants", Elektrotechnik \& Informationstechnik (2008) 125/12 pp. 409-414.

[2] F. A.Viawan, Karlsson D., "Combined Local and Remote Voltage and Reactive Power Control in the Presence of Induction Machine Distributed Generation", IEEE Power Systems Proceedings, Vol. 22, No.4, 2007, pp. 2003-2012.
[3] Bignucoloi, F., Caldon, R., Prandoni, V., Spelta, S., Vezzola, M., "The Voltage Control on MV Distribution Networks with Aggregated DG Units (VPP)," International Conference on the European Energy Market, EEM2009. IEEE International, 2009, pp. 1-6.

[4] M. R. Tousi, S. H. Hosseinian, M. B. Menhaj, "A Multi-Agent-Based Voltage Control in Power Systems Using Distributed Reinforcement Learning", McGraw Hill, 1980.

[5] J. S. Ravn, "A Multi-Agent Approach for Distribution System Restoration", Bachelors Degree Thesis, DTU Elektro Institut for Elektroteknologi, July 2008.

[6] S. Gehao, J. Xiuceng, Z. Yi, “Optimal Coordination For Multi-Agent Based Secondary Voltage Control In Power System”, 2005 IEEE/PES Transmission and Distribution Conference \& Exhibition: Asia and Pacific, Dalian, China, 2005.

[7] I. B. Mady, "Fuzzy Sets-Based Voltage Control Strategy for Radial Distribution Systems by Genetic Algorithm", 20th International Conference on Electricity Distribution, CIRED2009, Prague, June 2009. 